\title{
A proposed solution to the determination of water saturation: using a modelled equation
}

\author{
Jethro Sam-Marcus ${ }^{1} \cdot$ Efeoghene Enaworu $^{1}$ - Oluwatosin J. Rotimi ${ }^{1} \cdot$ Ifeanyi Seteyeobot $^{1}$
}

Received: 23 June 2017 / Accepted: 24 February 2018 / Published online: 13 March 2018

(c) The Author(s) 2018

\begin{abstract}
Reservoir characterization is an important phase in oil and gas field development, which takes place during the appraisal phase of either a green field or a brown field upon which further development options are considered. Water saturation is a very important parameter in the general description of the reservoir as well as equity determination and dynamic modelling. Numerous equations have been developed which have been used to determine water saturation, but calculated water saturation values have been inconsistent with the saturation values determined from core analysis. This is generally due to their inability to account for the varying distribution of shale in the reservoir and the often incorrectness of their underlying assumptions. The major aim of this research is to develop a model which can be used to determine water saturation values using data from well logs; also, to compare the developed model with other existing models used in the oil and gas industry, using data from core analysis and well logs as the input data; and then finally, to discuss the results of the comparison, using the core-derived saturation values as the bench mark. The model is based on a parallel resistivity model, which is based on the assumption that the conductivity of the sandstone term and the shale term exist in parallel in the shaly-sand reservoir. The shale term in the reservoir of the model is based on the assumption that the clay-bound electrons do not move in the same conductivity path as the sandstone electrons. The shale conductivity term is based on the bound water saturation and the bound water resistivity. The modelled equation was compared in two scenarios using well log data and core data from two different reservoirs, and the model showed consistency in predicting the average water saturation in both reservoirs. The results of the comparison were positive for the modelled equation, as it gave coherent results in both comparison scenarios and matched reasonably the average water saturation of the selected reservoirs. This developed model can serve as an accurate means of determining water saturation in reservoirs, especially for reservoirs with similar characteristics as the selected reservoirs in this research.
\end{abstract}

Keywords Reservoir characterization · Water saturation - Bound water saturation · Bound water resistivity · Volume of shale $\cdot$ Core analysis

\section{List of symbols}

$R_{\mathrm{o}} \quad$ Resistivity of the reservoir rock fully saturated with water

$R_{\mathrm{w}} \quad$ Resistivity of the formation water

a Tortuosity factor

$m \quad$ Cementation exponent

$\phi \quad$ Porosity

$F \quad$ Formation factor

$F^{*} \quad$ Formation factor for shaly sands

$Q_{\mathrm{v}} \quad$ Effective concentration of clay counterions

Jethro Sam-Marcus

jethro.sammarcus@gmail.com

1 Petroleum Engineering Department, Covenant University, Ota, Nigeria
$B \quad$ Equivalent conductance of clay counterions

$V_{\text {sh }} \quad$ Volume of shale

$m_{\text {sh }} \quad$ Shale cementation exponent

$\Phi_{\mathrm{t}} \quad$ Total porosity

$\Phi_{\text {tsh }} \quad$ Shale porosity

$F_{\text {sh }} \quad$ Shale formation resistivity factor

$R_{\mathrm{b}} \quad$ Bound water resistivity

$S_{\mathrm{b}} \quad$ Bound water saturation

\section{Introduction}

Reservoir characterization has been a very important tool in hydrocarbon exploration. It has enabled petroleum engineers to have a better understanding of the reservoir and 
its properties. Due to this fact, various models have been built to represent the reservoir and predict how the reservoir will behave under various conditions. Water saturation is an important parameter used in reservoir modelling, as it gives an idea of the percentage of the pore spaces occupied by water and oil or gas and hence the total amount of hydrocarbon present in the pore spaces of the reservoir.

The values of water saturation calculated for a particular reservoir are used as inputs to static models and dynamic models, and this in turn is used to determine the initial oil in place of a reservoir. The calculated values of oil in place form the basis of future production forecasts and the determination of the economic viability of the discovered reservoir. Therefore, high accuracy is needed in the determination of water saturation as it determines the oil in place and the estimated reserves.

Resistivity logs have been consistently used to determine the saturation of water in the reservoirs by making use of the Archie's equation (Archie 1942) that shows a relationship between water saturation to the true permeable formation resistivity, the formation porosity and the formation water resistivity. The challenge therefore arises due to the presence of shale in the reservoir which is a conductive medium and hence is against the original assumptions of the Archie's equation, which was a clean sandstone reservoir (Archie 1942). The presence of shale causes a disparity in the reading of the total resistivity of the reservoir and brings about an overshot in the water saturation predicted by the Archie's equation (Archie 1942). This disparity is caused by the additional conductance path caused by shale, and this additional conductance path is due to the conductive nature of shale.

Due to the economic importance of developing a model which would determine water saturation effectively with the highest form of accuracy, various models have been developed in order to take cognizance of the effect of shale on the overall reservoir resistivity as well as on the water saturation value determined for the reservoir. Various models such as the Simandoux equation (Simandoux 1963), the dual-water model (Clavier et al. 1977), the Waxman-Smits equation (Waxman and Smits 1968), Schlumberger equation (Schlumberger 1989), Indonesia model (Poupon and Leveaux 1971) were all built on the foundational idea presented by (Archie 1942) in his original paper, by including a shale factor into the original Archie equation and hence presenting a simpler way to determine water saturation. The simple equations run the risk of becoming too simple, but yet it has been noted that these equations are comprehensive and can perform very well when correctly applied depending on the aforedetermined properties of the reservoir. Yet there are more complex equations which are better functionally represented but contain values which are difficult to estimate accurately, which introduces a lot of errors in the calculated values of water saturation (Doveton 1986).
The gold standard of reservoir characterization has been chosen to be core analysis as it brings a representative sample of the reservoir to the laboratory where various properties can be determined from core analysis. Core analysis is very expensive and often inaccurate in representing the entire reservoir as only sections of the reservoir are taken to the laboratory and analysed (Odizu-Abangwu et al. 2010). Hence, more accurate equations need to be developed for various petroleum-producing regions as the geology of various regions is not the same.

This paper seeks to proffer a solution to the above-stated challenges by proposing a model as a possible solution to the shaly-sand problem.

\section{Literature review}

Since the advent of well logging, resistivity logs have been constantly used to determine the value of water saturation by using the Archie equation as the primary equation to determine water saturation. Due to the expensive nature of core analysis, the "log-only" option of determining water saturation has been seen as economical and truly desired (Doveton 1986).

The presence of shale, which is made up predominantly of clay minerals and silts, poses a major flaw to the Archie equation (Eq. 1), being that Archie assumed the reservoir was made up of purely sand and the only conductive medium was the reservoir water that saturated the reservoir rock (Archie 1942).

Based on this problem, shaly-sand equations have been developed to further account for the extra conductivity added to the total reservoir conductivity and invariably account for the shale effect and accurately determine the value of water saturation in the reservoir.

$S_{\mathrm{w}}=\sqrt[n]{\frac{a R_{\mathrm{w}}}{\emptyset^{m} R_{\mathrm{t}}}}$.

\section{Some shale sand models and their limitations}

Shaly-sand models would be considered and reviewed in this research as a premise into which Archie's model was modified. The shaly-sand models considered are:

- The Simandoux Equation

- The Schlumberger modification of the Simandoux equation

- Indonesia equation.

- The Waxman-Smits equation

- The dual-water model. 


\section{Simandoux equation}

Prior to the development of the Simandoux equation, the relationship between the true resistivity of the reservoir and the value of water saturation is represented in Eq. 2 .

$\frac{1}{R_{\mathrm{t}}}=\alpha S_{\mathrm{w}}+\frac{\beta}{R_{\mathrm{w}}} S_{\mathrm{w}}^{2}$

where $R_{\mathrm{t}}$ is true resistivity of the formation; $R_{\mathrm{w}}$, formation water resistivity; $S_{\mathrm{w}}$, water saturation; $\alpha$, shale term; $\beta$, sandstone term.

Simandoux in his experiment in 1963 studied "homogeneous mixtures of sorted sand and natural clay in various proportions." This was in order to study the volumetric effects of reducing clay volumes on the conductivity of the rock matrix and the overall saturation of water in the reservoir.

Hence, the Simandoux equation was presented as thus

$\frac{1}{R_{\mathrm{t}}}=\frac{S_{\mathrm{w}}^{2}}{F R_{\mathrm{w}}}+\frac{V_{\mathrm{sh}} \varepsilon}{R_{\mathrm{sh}}}$

With its shale term dependent on $V_{\mathrm{sh}}$ (volume of shale) and $R_{\mathrm{sh}}$ (resistivity of shale). The Simandoux equation was later modified by Bardon and Pied (1969) by including water saturation to the shale term of the original Simandoux equation which turned Eq. 3 into Eq. 4.

$\frac{1}{R_{\mathrm{t}}}=\frac{S_{\mathrm{w}}^{2}}{F R_{\mathrm{w}}}+\frac{V_{\mathrm{sh}} * S_{\mathrm{w}}}{R_{\mathrm{sh}}}$

Some of the notable short-comings of the Simandoux equation were as follows (Herrick and Kennedy 2009).

Simandoux made measurements on only four synthetic samples using one type of clay (montmorillonite), and the samples used had a constant value of porosity. Other researchers have demonstrated that the shale effect $\left(\alpha=\frac{V_{\mathrm{sh}}}{R_{\mathrm{sh}}}\right)$ does not apply to disseminated shale conditions. The Simandoux model leads to optimistic results when the porosity is less than $20 \%$, and because of this fact, it cannot be relied on in low porosity situations. The first terms of the Simandoux equations do not show a volumetric balance between the sandstone volume and the clay volumes, and the lack of a shale formation factor in the clay term makes the correction for clay effect by the Simandoux equation too large and hence reduces the amount of water saturation calculated. This sole problem could lead to the overestimation of the quantity of hydrocarbons in place. Schlumberger modified the general Simandoux equation by adding $1-V_{\mathrm{sh}}$ to the denominator to account for the shaly nature inherent in the clean sands. Hence, Eq. 5 is

$\frac{1}{R_{\mathrm{t}}}=\frac{S_{\mathrm{w}}^{2}}{F\left(1-V_{\mathrm{sh}}\right) R_{\mathrm{w}}}+\frac{V_{\mathrm{sh}}}{R_{\mathrm{sh}}} S_{\mathrm{w}}$
The argument was that the original Simandoux equation completely discarded the possibility of having shale within the clean sandstone layers. This modification was done by Schlumbeger in their paper as a crude way to calibrate their equipment without any actual physical basis for this addition. It was their means of correcting the errors included in the Simandoux equation when the formation resistivity factor of clay was not accounted for in the shale term (Schlumberger 1989).

The first use of the $1-V_{\mathrm{sh}}$ term was by Poupon et al. (1954), which was based on a volumetric balance between the volume of shale present and the volume of clay present in the reservoir. It was used to determine the volume of water saturation in thin-bedded sands and shale. It basically assumes that the conductivity of a particular medium is based on its size and the conductive material within its pore spaces (Eq. 6).

It is represented by this equation:

$\frac{1}{R_{\mathrm{t}}}=\frac{\left(1-V_{\mathrm{sh}}\right) S_{\mathrm{w}}^{n}}{F R_{\mathrm{w}}}+\frac{V_{\mathrm{sh}}}{R_{\mathrm{sh}}}$

\section{Indonesia equation}

Poupon and Leveaux (1971) derived the Indonesian equation in order to account for the high mount of shale and fresh water formations, which is common in Indonesia reservoirs. The equation was developed by using computer-made crossplots to determine the relationship between the value of water saturation and the value of the true resistivity of the formation. The range of shale volumes recorded for such formations was $30 \%-70 \%$ shale content

$\frac{1}{\sqrt{R_{\mathrm{t}}}}=\left[\frac{V_{\text {clay }}^{d}}{\sqrt{R_{\text {clay }}}}+\frac{\emptyset^{m / 2}}{\sqrt{a R_{\mathrm{w}}}}\right] S_{\mathrm{w}}^{n / 2}$

where $V_{\text {clay }}$ is volume of shale; $R_{\mathrm{t}}$, formation true resistivity; $R_{\mathrm{w}}$, formation water resistivity; $a$, tortuosity, $\phi$, porosity; $S_{\mathrm{w}}$, water saturation

$d=1-\frac{V_{\mathrm{sh}}}{2}$

\section{Waxman-Smits equation}

Waxman-Smits proposed a model which was based on the understanding that "one" water (saturating brine) was present in the reservoir (Waxman and Smits 1968). The Waxman-Smits model is based on laboratory measurements of resistivity, porosity and saturation of real rocks, and due to the model being backed-up heavily by laboratory data, the model was generally accepted (Eq. 8).

$\frac{1}{R_{\mathrm{t}}}=\frac{S_{\mathrm{w}}^{2}}{F^{*} R_{\mathrm{w}}}+\frac{B Q_{\mathrm{v}} S_{\mathrm{w}}}{F^{*}}$ 
where $F^{*}$ is formation factor for shaly sands; $Q_{\mathrm{v}}$, effective concentration of clay counterions; $B$, equivalent conductance of clay counterions.

The major assumptions of the Waxman-Smits model about clay formation and its properties are as follows:

Clay surface conductivity is assumed to share a directly proportional relationship with the factor $Q_{\mathrm{v}}$ (defined as the milli-equivalents of exchangeable clay counterions per unit volume of pore space). The constant of proportionality in this relationship was referred to as $\mathrm{B}$, which is defined as the equivalent conductance of the clay counterions. The Waxman-Smits equation assumes that "the electric current is transported by the clay counterions that travels along the same tortuous paths as the current attributed to ions in the pore water" (Waxman and Smits 1968).

The second assumption of the Waxman-Smits model is the major reason for the $F^{*}$ term replicated in both the sandstone resistivity term and the shale resistivity term. Hence, the shale term and the sandstone term are seen to have them same formation resistivity factor (Herrick and Kennedy 2009).

This model served as the premise of the widely used dualwater model. The Waxman-Smits equation is often used as a standard against other methods, due to its high experimental backing, but the determination of CEC (Cation Exchange Capacity) is a time-consuming experiment and this is the major limitation of the Waxman-Smits model.

\section{Dual-water model}

In the dual-water model, it is proposed that the impact of clay minerals on the resistivity of reservoir rock is caused by the presence of two waters in the reservoir: the free water within the pore spaces of the reservoir rock and the bound water within the clay matrix (Clavier et al. 1977). The dualwater model was developed with the basic aim of accounting for the conduction that occurs within the volume at the surface of the clay mineral. The idea was to account for the conductivity that occurs near and within the double layer and the conductivity that occurs in the layer free from the effects of clay. Though the dual-water model was developed with the aim of modifying the Waxman-Smits model for water saturation, it contains within itself the premise that the conduction geometry of the free water and the clay counterions is the same (Herrick and Kennedy 2009).

The dual-water model is represented by Eq. (9).

$\frac{1}{R_{\mathrm{t}}}=\frac{S_{\mathrm{w}_{\mathrm{t}}}^{n}}{F_{\mathrm{o}}}\left[\frac{1}{R_{\mathrm{w}}}+\frac{V_{\mathrm{Q}} Q_{\mathrm{V}}}{S_{\mathrm{w}_{\mathrm{T}}}}\left(\frac{1}{R_{\mathrm{cw}}}-\frac{1}{R_{\mathrm{w}}}\right)\right]$

where $R_{\mathrm{cw}}$ is resistivity of the bound water; $R_{\mathrm{w}}$, resistivity of the free water.

\section{Methodology}

The model developed in this paper is based on a parallel conductivity model which states that the total conductivity of the formation is a combination of the conductivity of the formation water in parallel with the conductivity contribution of the clay term. Hence, the formation water conducts in series with the clay itself and the clay-bound water.

This model also refutes the assumption of the Waxman-Smits and dual-water model which believe that the formation water and the clay counterions all flow through the same tortuous path and hence have the same formation resistivity factor.

The modelled equation is represented in terms of resistivity mathematically as:

$\frac{1}{R_{\mathrm{t}}}=\frac{1}{R_{\mathrm{ss}}}+\frac{1}{R_{\mathrm{sh}}}$

where $R_{\mathrm{t}}$ is true resistivity; $R_{\mathrm{ss}}$, sandstone resistivity contribution; $R_{\mathrm{sh}}$, shale resistivity contribution

$\frac{1}{R_{\mathrm{ss}}}=\frac{S_{\mathrm{w}}^{n}}{F * R_{\mathrm{w}}}$

The resistivity in the sandstone term is the same as the Archie's resistivity equation, with the major contributor to the conductivity of the sandstone reservoir being the formation water resistivity. The shale bound water saturation itself is modelled to be a part of the total water saturation and is seen as the linking term between the " $V_{\text {sh }}$ models" and the "CEC" cation-ion exchange capacity models.

The bound water saturation is given by:

$S_{\mathrm{b}}=\frac{V_{\mathrm{sh}} \emptyset_{\mathrm{tsh}}}{\emptyset_{\mathrm{t}}}$.

The bound water resistivity is given by:

$R_{\mathrm{b}}=R_{\mathrm{sh}} \emptyset_{\mathrm{tsh}}^{\mathrm{msh}}$

where $m_{\mathrm{sh}}$ is shale cementation exponent; $\Phi_{\mathrm{t}}$, total porosity; $\Phi_{\text {tsh }}$, shale porosity.

The shale formation factor then becomes:

$\mathrm{F}_{\mathrm{sh}}=\frac{1}{\emptyset_{\mathrm{tsh}}^{\mathrm{msh}}}$

Replacing the $V_{\mathrm{sh}}$ term with $S_{\mathrm{b}}$, the $R_{\mathrm{sh}}$ term with $R_{\mathrm{b}}$ and including the $F_{\mathrm{sh}}$ term (Shale formation volume factor) in the Simandoux equation, Eq. (3) then becomes Eq. (15):

$\frac{1}{R_{\mathrm{t}}}=\frac{S_{\mathrm{w}}^{n}}{F R_{\mathrm{w}}}+\frac{S_{\mathrm{b}} S_{\mathrm{w}}^{(n-1)}}{F_{\mathrm{sh}} R_{\mathrm{b}}}$.

This model was developed based on the following assumptions: 
1. Parallel conductivity exists between the clean sand and the shale present in the clean sand. The sum of all parallel conductivity is equal to the total reservoir conductivity.

2. The shale term and the sandstone term do not have the same formation resistivity factor.

3. In the cases of thin-bedded shale and sandstone reservoirs, a volumetric balance exists in the reservoir such that the volumetric concentration of the sands summed up with the volumetric concentrations of the clay is equal to unity.

4. The total shale resistivity is a function of the clay-bound water saturation, its resistivity and the shale formation factor.

Table 1 Reservoir properties

\begin{tabular}{ll}
\hline Reservoir properties & Value \\
\hline$a$ & 1 \\
$m$ & 1.86 \\
$n$ & 2.2 \\
$R_{\mathrm{w}}$ & 0.06771 \\
$R_{\mathrm{sh}}$ & 2 \\
$\Phi_{\mathrm{tsh}}$ & 0.077 \\
\hline
\end{tabular}

\section{Results}

The model was tested using a reservoir that was divided into two zones. The first reservoir zone had high shale content, while the second zone mimicked the Archie clean sand, with very low volumes of shale. The selected reservoir properties used for the analysis are recorded in Table 1. Figure 1 shows the overall reservoir trend for the various values of water saturation calculated from each of the selected model. The trend between the core data water saturation and the results of the developed model is shown in Fig. 2.

To further run an analysis on the results, a comparison between the values of average water saturation derived from each model was done, and the equation with a value of average water saturation that best matched the cored values of water saturation was selected based on the analysis. The analysis was broken down into two parts, the first part being a comparison of the average water saturation values from each equation for the entire reservoir as shown in Table 2 and the second part being the calculation of the values of average water saturation from each equation, for each reservoir zones as presented in Tables 3 and 4.

It was observed that the average water saturation values from the modelled equation were very close to already established equations. The Archie equation had the least accuracy in both comparisons due to its lack of a shale term. The average water saturation of the modelled equation was close to the average water saturation from the cored data.

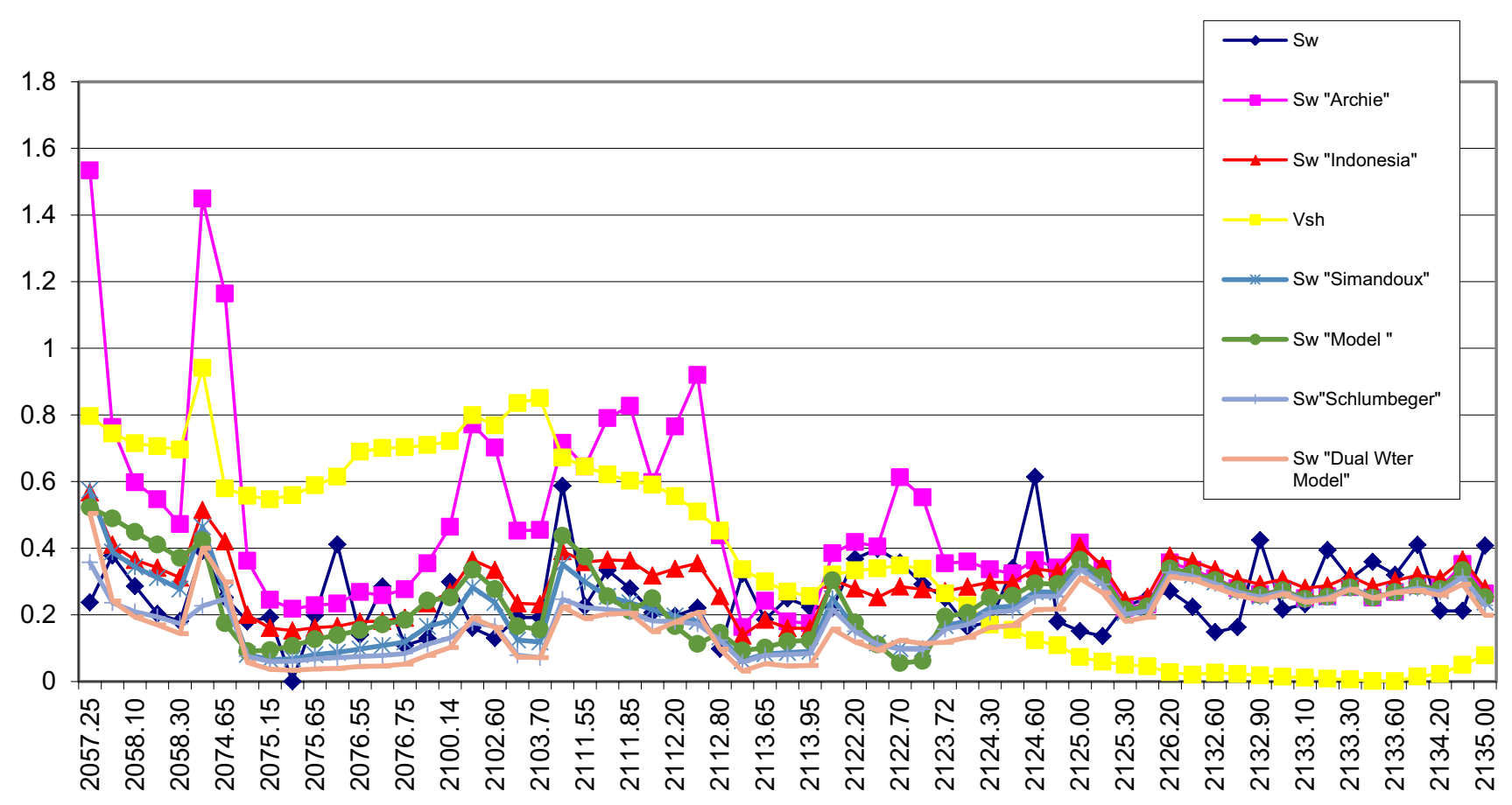

Fig. 1 Water saturation trend with varying values of volume of shale in the reservoir 


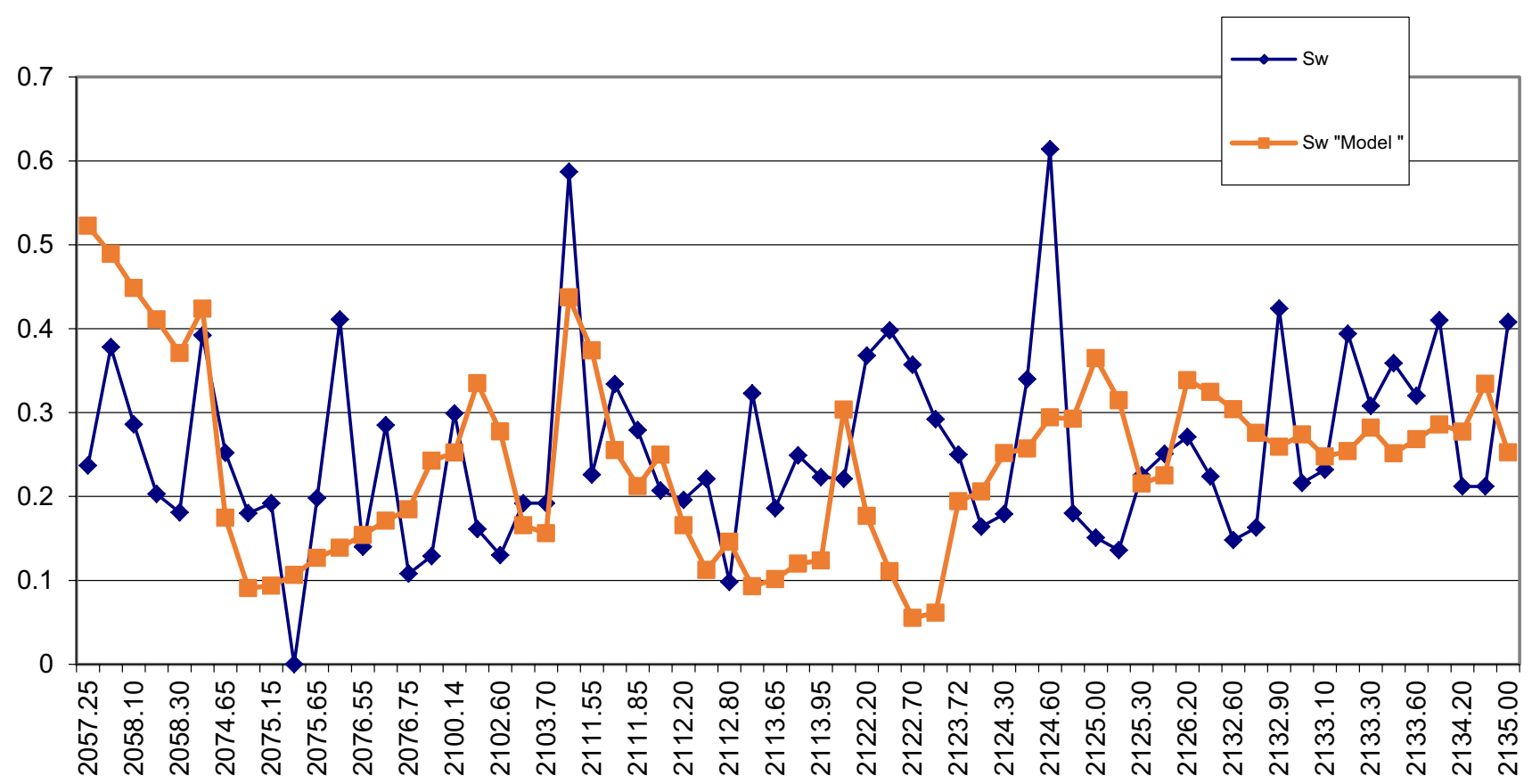

Fig. 2 Trend comparison between modelled equations and the core water saturation

Table 2 Overall reservoir water

Table 3 Water saturation analysis for Zone 1 saturation values

\begin{tabular}{lll}
\hline Overall $S_{\mathrm{w}}$ & & \\
\hline$S_{\mathrm{w}}$ & 0.295 & 29.5 \\
Archie & 0.552 & 55.2 \\
Indonesia & 0.321 & 32.1 \\
Simandoux & 0.252 & 25.2 \\
Schlumberger & 0.194 & 19.4 \\
DWM & 0.185 & 18.5 \\
Model & 0.292 & 29.2 \\
\hline
\end{tabular}

\begin{tabular}{lrr}
\hline Zone 1 & & \\
\hline$S_{\mathrm{w}}$ & 0.30 & 30.34 \\
Archie & 1.05 & 105.25 \\
Indonesia & 0.41 & 40.79 \\
Simandoux & 0.33 & 33.25 \\
Schlumberger & 0.20 & 19.96 \\
DWM & 0.28 & 28.39 \\
Model & 0.31 & 31.43 \\
\hline
\end{tabular}

A second comparison was done in order to test the consistency of the derived model. The reservoir volume of shale values ranged from $10 \%$ to $70 \%$. Table 5 shows the reservoir properties used in the second comparison.

On an average, the values of water saturation calculated using the thickness-weighted average technique were
Table 4 Water saturation analysis for Zone 2

Table 5 Reservoir properties

\begin{tabular}{ll}
\hline Reservoir properties & Values \\
\hline$a$ & 1 \\
$m$ & 1.96 \\
$n$ & 2 \\
$R_{\mathrm{w}}$ & 0.031 \\
$R_{\mathrm{sh}}$ & 2.9 \\
$\Phi_{\mathrm{tsh}}$ & 0.0614 \\
\hline
\end{tabular}

consistent for the derived model as presented in Table 6 . However, the derived model is perceived as the most promising model and this is due to its ability to account for the bound water saturation, the bound water resistivity and the conductive path of the bound water electrons; all these were taken into consideration during the development of this model. 
Table 6 Average water saturation for the selected reservoir
Average reservoir water saturation

\begin{tabular}{ll}
\hline$S_{\mathrm{w}}$ "core data" & 20.58 \\
$S_{\mathrm{w}}$ "Schlumberger" & 16.05 \\
$S_{\mathrm{w}}$ "Simandoux" & 17.36 \\
$S_{\mathrm{w}}$ "dual-water model" & 14.28 \\
$S_{\mathrm{w}}$ "Model" & 20.02 \\
\hline
\end{tabular}

\section{Conclusion}

The major aim of this research was to propose a solution to the shaly-sand problem by developing a model which could accurately mirror the water saturation results from core analysis and have average water saturation values which are not too far from the values of average water saturation gotten from core analysis. A solution has been proposed, the model is a physical model whose mathematical relation was based on the relationship between the formation conductivity and the bound water saturation and the bound water resistivity. Its major assumption was that the clay-bound electrons and the sandstone electrons do not move through the same conductive path. The results from the model were coherent with the results of average water saturation in each comparison cases.

The conclusion can be reminisced in three points:

1. The derived model was consistent in both comparison cases. The derived model was the most consistent equation in both cases of water saturation comparison, with average water saturation values being close to those from core analysis.

2. Accounting for the bound water saturation, the bound water resistivity, and the assumption that the claybound electrons and the sandstone electrons do not flow through the same conductive path is the one of the main reasons why the modelled equation was able to effectively mirror the average reservoir water saturation.

3. The modelled equation was consistent in the all the cases of comparison. It shows promise and should be further tested and applied to more oil fields.

Acknowledgements The author is grateful to the management of Covenant University for permission to publish this paper.

Open Access This article is distributed under the terms of the Creative Commons Attribution 4.0 International License (http://creativeco mmons.org/licenses/by/4.0/), which permits unrestricted use, distribution, and reproduction in any medium, provided you give appropriate credit to the original author(s) and the source, provide a link to the Creative Commons license, and indicate if changes were made.

\section{References}

Archie GE (1942) The electrical resistivity log as an aid in determining some reservoir characteristics. Trans AIME 146:54-62

Bardon Ch, Peid B (1969) Formation water saturation in shaly sands. SPWLA 10th annual logging symposium, 25-28 May, Houston, Texas

Clavier C, Coates G, Dumanoir J (1977) The theoretical and experimental bases for the "dual water" model for the interpretation of shaly sands. In: Society of Petroleum Engineers Paper No. 6859, p 10

Doveton JH (1986) Log analysis of subsurface geology: concepts and computer methods, 1st edn. Wiley, New York, NY, p 273

Herrick D, Kennedy W (2009) On the quagmire of "Shaly Sand" saturation equation. In: Society of petrophysicists and well log analysts, The Woodlands, Texas, United States, pp. 1-16

Odizu-Abangwu I, Suleman A, Nwosu C (2010) The impact of different shaly sand models on in place volumes and reservoir producibility in Niger Delta reservoirs. The Dual water and the Normalized Waxman-Smith saturation models. In: SPE 140627. Society of Petroleum Engineers, Tinapa-Calabar, Nigeria, p 6

Poupon A, Leveaux J (1971) Evaluation of water saturation in shaly formations. In: SPWLA 12th annual logging symposium, pp 1-2

Poupon A, Loy ME, Tixier MP (1954) A contribution to electric log interpretation in shaly sands. Trans AIME 6(06):138-145

Schlumberger (1989) Log interpretation-principles! Applications. In: Schlumberger Educational Services, pp 8-14

Simandoux P (1963) Measures die techniques an milieu application a measure des saturation en eau, etude du comportement de massifs agrileux. Review du'Institute Francais du Patrole 18(Supplementary Issue): 193

Waxman MH, Smits LJ (1968) Electrical conductivities in oil-bearing shaly sands. In: Society of petroleum engineers $42 \mathrm{nd}$ annual fall meeting. Society of Petroleum Engineers, Houston, Texas, pp 107-122

Publisher's Note Springer Nature remains neutral with regard to jurisdictional claims in published maps and institutional affiliations. 